\title{
Erfolgreich ins Management einsteigen
}

\author{
Ergebnisse der Absolventenbefragung eines Masterstudiengangs \\ »Sozialmanagement «
}

Armin Wöhrle

\begin{abstract}
Die Übernahme von Leitungsfunktionen in sozialen Organisationen verlangt neben den fachlichen Kenntnissen zusätzliches rechtliches und betriebswirtschaftliches Wissen. Gefragt sind zudem soziale Schlüsselqualifikationen, um eine Führungsrolle reflektieren und ausfüllen zu können. Ein Zusatzstudium kann dabei belfen, wie ein Beispiel in Sachsen zeigt.
\end{abstract}

Wenn die Entwicklungen nicht so vorangehen, wie man sich es wünscht, dann möchte man schon gern an den Stellschrauben drehen. Wenn man nicht schon nach dem Rentenalter schielt, sondern noch etwas bewegen will, so kommt über die Fortbildung hinaus die Weiterqualifikation in den Blick, mit der man in Leitungsfunktionen aufsteigen kann. Mit dem Bolognaprozess und der Vorstellung des lebenslangen Lernens gibt es für Sozialarbeiterinnen und Sozialarbeiter, für Sozialpädagoginnen und Sozialpädagogen die Möglichkeit, berufsbegleitend einen Masterabschluss zu erwerben, der für das Sozialmanagement qualifiziert. Doch ermöglicht er wirklich ein berufliches Fortkommen?

Eine Untersuchung an der Hochschule Mittweida, die seit 1999 berufsbegleitend in einem Masterstudiengang Sozialmanagement ausbildet, gibt Aufschluss über die Meinungen ihrer Absolventen und Absolventinnen. Es wurden alle Absolventen mittels eines umfangreichen Fragebogens befragt. Die Rücklaufquote ist mit 45,7 Prozent der noch erreichbaren ehemaligen Studierenden (bzw. 38,2\% aller Absolventen) erfreulich hoch.

Prof. Dr. Armin Wöhrle lehrt

Sozialmanagement,

Projektentwicklung und Beratung an

der Hochschule Mittweida in

Sachsen.

E-Mail woehrle@htwm.de
In den Matrikeln 1999 bis 2003 haben 76 Teilnehmerinnen und Teilnehmer das berufsbegleitende Studium aufgenommen und 55 Studierende beendeten es bis Ende 2006 erfolgreich. Die niedrige Zahl des Durchlaufs erklärt sich aus der Aufnahme geringer Gruppengrößen, die entsprechend gut in Präsenzeinheiten mit Trainings und Coaching betreut werden können. Es muss kaum gesagt werden, dass es sich für den Personenkreis aus der Praxis um eine intensive Kraftanstrengung handelte, »nebenbei « ein Masterstudium auf hohem Niveau zu absolvieren.

Die Untersuchung filterte verschiedene Kennzeichen der Personengruppe heraus. So ist die Mehrzahl der Befragten zwi-
Formulierungen, wie »Gerade noch machbar « oder »Anstrengend, jedoch nicht unmöglich «. Etwa zehn Prozent der Befragten äußerten Bedenken hinsichtlich der Menge der zu bearbeitenden Studienbriefe und des Umfangs der Masterarbeit. 19 Prozent beantworteten diese offen formulierte Frage nicht.

Trotz aller Belastungen würden sich 91 Prozent der Absolventinnen und Absolventen wieder für den Studiengang Sozialmanagement der Hochschule Mittweida entscheiden. Insbesondere hervorgehoben wurden bei der Bewertung:

- die Kompetenz der Dozenten

- der gute Kontakt zwischen Studierenden und Dozenten

\section{"Ein Studium neben dem Beruf kostet Kraft, lässt aber noch Zeit für soziale Kontakte«}

schen Anfang 30 und Ende 40, wobei die Mitte-40-Jährigen besonders stark vertreten sind. Sowohl bei den Studienanfängerinnen (41) und Studienanfänger (35) als auch bei den Absolventinnen (29) und Absolventen (26) sind Frauen stärker vertreten. Zwei Drittel der Studierenden lebte sowohl zum Studienbeginn als auch Zeitpunkt der Befragung in einer Partnerschaft und 58 Prozent der Befragten haben Kinder. Es ist also - trotz aller Kraftanstrengungen, die erfordert werden keine Unternehmung, für die man sich aller sozialen Bande entledigen muss.

Ebenso wurde die Belastung durch das Studium erfragt. Ein Drittel der Absolventinnen und Absolventen schätzte das Studium rückblickend als gut bis sehr gut vereinbar mit ihrer beruflichen Tätigkeit ein. Weitere 38 Prozent antworteten auf die Frage »Wie empfanden Sie die Vereinbarkeit des Studiums mit Ihrer beruflichen Tätigkeit? « mit einschränkenden
- die Qualität der Studienbriefe

- das Coaching

- die Qualität der Präsenzeinheiten

- die Praxisnähe der Lehrinhalte sowie - die fachliche Qualität des Studiums.

Die Nützlichkeit des Studiums wird durch die Untersuchung bestätigt: 95 Prozent der Befragten beantworteten die offene Frage: »Wie bewerten Sie die Nützlichkeit Ihres Studiums aus heutiger Sicht? « und durchweg alle Antworten heben positive Aspekte hervor. Die Einschätzungen reichen von »sehr nützlich «, "sehr hilfreich « über »Ich habe insgesamt sehr davon profitiert « bis zu »Die erworbenen Kenntnisse werden fast täglich angewendet «.

71 Prozent der Befragten nannten explizit die positive Bedeutung des Studiums für die eigene berufliche Entwicklung. 48 Prozent der Absolventinnen und Absolventen betonten die Rolle des Studiums für ihre Persönlichkeitsentwik- 
klung. 24 Prozent heben ihre gestiegenen Chancen auf dem Arbeitsmarkt hervor. Ebenfalls 24 Prozent kritisieren den geringen Bekanntheitsgrad des Abschlusses auf dem Arbeitsmarkt.

Konkret nachgefragt, wie sich das Studium rechnet, erbrachte die Studie, dass 43 Prozent der Befragten seit Studienbeginn ein- bis zweimal ihr Anstellungsverhältnis wechselten. 24 Prozent derer, die noch nicht in Leitungs- und Führungspositionen waren, übernahmen danach solche und 43 Prozent der Befragten verdienen deutlich mehr als zu Studienbeginn (Steigerungen insbesondere bei 4.000 Euro brutto und darüber).

Für die weitere Perspektive begrüßen zwei Drittel der Absolventinnen und Absolventen die Möglichkeit, im Anschluss an das Sozialmanagementstudium promovieren zu können. Sechs Teilnehmende äußerten in Abhängigkeit von der zeitlichen Vereinbarkeit mit Beruf und Familie, der finanziellen Realisierung und formalen Fragen zum Ablauf des Promotionsvorhabens persönlich großes Interesse an einer Promotion.

\section{Information}

Das Studium des Sozialmanagements an der Hochschule Mittweida (FH) soll Absolventen eines ersten berufsqualifizierenden Studiums dazu befähigen, Managementfunktionen in allen Bereichen der Sozialwirtschaft, beispielsweise in der freien Wohlfahrtspflege, bei öffentlichen Trägern und in privatwirtschaftlichen Organisationen, sowie - mit entsprechenden selbstständigen Vertiefungen - in angrenzenden Bereichen des Gesundheits-, Bildungs-, Kultur- und öffentlichen Sektors kompetent auszuüben. Der Masterstudiengang Sozialmanagement bietet eine interdisziplinäre Ausbildung für Führungs- und Leitungsfunktionen auf der Basis einer praxisbezogenen Verknüpfung von relevantem Orientierungs-, Erklärungs-, Analyse- und Handlungswissen der Wissenschaftsdisziplinen Soziale Arbeit, Betriebswirtschaftlehre, Soziologie, Psychologie, Sozialpolitik und Recht.

Angaben zum Studiengang und zur Befragung finden sich auf der Website der Hochschule Mittweida:

Internet http://www.htwm.de/sa.

\section{Themenhefte der Blätter der Wohlfahrtspflege}

Der Allgemeine Sozialdienst

Blätter der Wohlfahrtspflege 1/2002

Lebensführung, Lebensbewältigung

Blätter der Wohlfahrtspflege 2/2002

Daseinsvorsorge

Extra: Die PISA-Studie und die

Bürgergesellschaft

Blätter der Woblfahrtspflege 3/2002

Armut und Integration

Blätter der Wohlfahrtspflege 4/2002

Lebenslagen im Alter

Blätter der Wohlfahrtspflege 5/2002

Bürgerbeteiligung

Blätter der Woblfahrtspflege 6/2002

Bildung und Soziale Arbeit

Blätter der Wohlfahrtspflege 1/2003

Menschen mit Behinderungen

Blätter der Wohlfahrtspflege 2/2003

Familienpolitik, Familienhilfe

Blätter der Wohlfahrtspflege 3/2003

Fachsozialarbeit

Blätter der Wohlfahrtspflege 4/2003

Unternehmensführung in der Altenhilfe

Blätter der Woblfahrtspflege 5/2003

Elementarerziehung

Blätter der Woblfahrtspflege 6/2003

Kommunale Sozialpolitik

Blätter der Woblfahrtspflege 1/2004

Case Management

Blätter der Wohlfahrtspflege 2/2004

Brennpunkte der Jugendhilfe

Blätter der Woblfahrtspflege 3/2004

Ambulante Pflege

Blätter der Wohlfahrtspflege 5/2004

Interkulturelle Arbeit

Blätter der Woblfahrtspflege 6/2004

Demografischer Wandel

Blätter der Wohlfahrtspflege 1/2005

Bildung

Blätter der Woblfahrtspflege 2/2005

Bürgerengagement

Blätter der Wohlfahrtspflege 3/2005

Gemeindepsychiatrie

Blätter der Wohlfahrtspflege 4/2005

Standards Sozialer Arbeit

Blätter der Wohlfahrtspflege 5/2005
Performanz - Wissen, was man tut, und zeigen, was man leistet Blätter der Woblfahrtspflege 6/2005

Wohngemeinschaften Blätter der Woblfahrtspflege 1/2006

Ausbildung in der Sozialen Arbeit Blätter der Woblfahrtspflege 2/2006

Die Verortung Sozialer Arbeit Blätter der Woblfahrtspflege 3/2006

Wirtschaft

Blätter der Woblfahrtspflege 4/2006

Das Persönliche Budget

Blätter der Woblfahrtspflege 5/2006

Engagement als Ressource

Blätter der Woblfahrtspflege 6/2006

Soziale Arbeit und Wohnungswirtschaft

Blätter der Wohlfahrtspflege 1/2007

Stiftungen

Blätter der Wohlfahrtspflege 2/2007

Beratung

Blätter der Wohlfahrtspflege 3/2007

Krisendienste

Blätter der Wohlfahrtspflege 4/2007

Schnittstellen der Jugendhilfe Blätter der Wohlfahrtspflege 5/2007

Selbständigkeit in der Sozialen Arbeit Blätter der Wohlfahrtspflege 6/2007

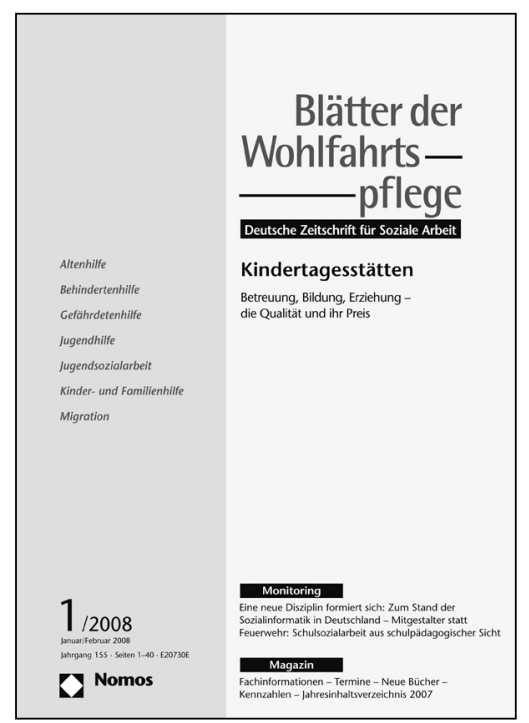

Kindertagesstätten

Blätter der Woblfahrtspflege 1/2008
Der Jahresbezugspreis beträgt 58,- Euro (für Studierende und arbeitslose Bezieher auf jährlichen Nachweis 29,- Euro). Die Einzelhefte können zum Preis von 11,- Euro zuzüglich Versandkosten und Mehrwertsteuer bezogen werden bei: Nomos Verlagsgesellschaft, 76520 Baden-Baden, Telefon 07221 2104-39, Fax 07221 210443, E-Mail hohmann@nomos.de 